\title{
Optimal investment in the energy system of Zero Emission Neighborhoods considering the refurbishment of the building stock.
}

\author{
Dimitri Pinel ${ }^{1 *}$, Magnus Kopås ${ }^{1}$ \\ ${ }^{1}$ NTNU, Department for Electric Power Engineering, 7491 Trondheim, Norway
}

\begin{abstract}
To increase the impact that Zero Emission Neighbourhoods (ZEN) can have in the effort to decrease $\mathrm{CO} 2$ emissions, the refurbishment of the existing building stock is a parameter that should be considered. The existing literature contains work on optimization models for the energy system of neighbourhoods taking into account emissions but fails to account for the refurbishment of buildings. This paper addresses this option and presents an optimization model for designing a cost-optimal energy system of a ZEN in the context of existing buildings. The model is presented and used in a case study in Norway and compared to a case with linearized binaries. A sensitivity analysis is performed on the cost of refurbishment. With the original refurbishment cost assumptions, it is not chosen by the optimization, contrary to the hydronic. The system relies mainly on PV, solar thermal collectors (ST), a biogas engine, a battery and heat pumps (HP) and heat storage. From $50 \%$ of the original refurbishment cost, it is chosen, and the system does not have a biogas engine and a heating grid anymore, but a much bigger battery and more heating technologies inside the buildings. With linearized binaries, the investments are similar to the case with $50 \%$ refurbishment cost, but the value of the linearized binaries cannot be used to indicate the share of building to refurbish.
\end{abstract}

\section{Introduction}

Neighborhoods and the energy use of their buildings represent an important share of the global emissions of carbon dioxide. Zero Emission Neighborhoods (ZEN) is a concept that aims at reducing their contribution to the emissions. In the research centre for Zero Emission Neighborhoods in smart cities (FME ZEN) in Norway, several disciplines collaborate towards making more sustainable cities ${ }^{\dagger}$. In this research centre, ZENs are defined as neighborhoods that reach net zero emission of $\mathrm{CO} 2$ over their lifetime. One of the problems arising is how to design the energy system of such a neighborhood to reach net zero emission target. ZENIT is an optimization model created for this purpose. This model finds the energy system design which satisfies the emission constraint at the lowest cost [1]. However, this model can only be used for greenfield development.

In order for the ZEN concept to have a bigger impact, it is important to modify the models to be able to account for existing buildings.

This paper aims at investigating one of the gaps in the research literature by addressing the inclusion of refurbishment of buildings in optimization models designing the energy system of ZEN and their impact on the result. This is important in order to facilitate existing buildings and neighborhoods to participate in emission reduction measures and facilitate the transition to a decarbonized future.

In this paper, a model for the investment in the energy system of ZEN is presented and the modifications done to take the refurbishment of buildings into account are introduced. The model is then used on the case of an existing neighbourhood in Norway and the results are discussed and compared to the case when the binaries for refurbishment and hydronics are linearized. A sensitivity analysis is performed on the cost of refurbishment.

\section{Literature review}

Several models to deal with the investment in the energy system of neighborhoods are presented in the literature [2] [3] [4] [5] [6] [1] [7]. Each model is a Mixed Integer Linear Program (MILP) but emphasizes different elements. For instance, [6] and [7] include the heating

\footnotetext{
* Corresponding author: dimitri.q.a.pinel@ntnu.no

$\dagger$ https://fmezen.no/
} 
grid layout as part of the problem. Seasonal storage is included in [4] and [1]. Voltage constraints and power flow are included in [5] while [3] focuses on combining open source models.

[8] simplifies the MILP optimization problem by separating it in parts and finds near optimal solutions.

[8] also addresses the refurbishment of neighborhoods envelope in the optimization. The impact of changing insulation thickness of windows on the annual heat demand is calculated based on a building standard and an equivalent insulation thickness is added as a variable in the optimization.

[9] takes existing buildings and compares different combination of retrofitting measures and energy system design. One specific feature of the study is the inclusion of façade PV. Cost assumptions for refurbishment as well as impact on annual loads are presented. They find that passive reductions of the loads are not profitable, even when assuming increasing $\mathrm{CO} 2$ prices impact on retail electricity prices.

On the other hand, there are models that do not deal with the energy system of neighborhoods but deal with optimising the envelope of buildings. [10] considers the optimization of the envelope of the building in a multiobjective metaheuristic algorithm to look for the optimal life cycle cost and emissions and finds a pareto front of solutions for the materials of the building envelope. Moreover, [11] also uses a multi-objective metaheuristic approach to find building parameters such as the building orientation, aspect ratio, windows, wall and roof type and materials.

Other models fall in between the two types of models presented earlier, with for example optimization of the building's material and windows and investment in PV panels such as [12].

In this paper, we introduce refurbishment into the problem of the design of the energy system of ZENs, but we do not decompose into subproblems and go to the level of detail of the envelope of the buildings.

We show a formulation of a model for the design of the energy system of a ZEN, considering the refurbishment and the hydronic system and their modelling implications. It contributes to the existing literature by introducing refurbishment in the context of ZEN's energy system.

\section{Model Presentation}

The ZENIT (Zero Emission Neighborhood Investment Tool) is presented in [1] and this section presents an extension accounting for refurbishment. The model minimizes the energy system investment and operation cost for a given neighborhood that allows to be zero emission in the lifetime using a MILP formulation. Clusters are used to reduce the computational complexity.

The objective function is to minimize the following expression (Eq. (1)).

In Eq. (1), $b^{H G}, b_{b}^{\text {refurb }}$ and $b_{b}^{\text {hyd }}$ are the binaries controlling the investment in the heating grid and the refurbishment and hydronic system of the building $b$.

$$
\begin{gathered}
b^{H G} \cdot C^{H G}+\sum_{b}\left(\sum _ { i } \left(\left(C_{i, b}^{\text {var,disc }}+\right.\right.\right. \\
\left.\left.\frac{C_{i, b}^{\text {maint }}}{\varepsilon_{r, D}^{\text {tot }}}\right) \cdot x_{i, b}+C_{i, b}^{\text {fix,disc }} \cdot b_{i, b}\right)+b_{b}^{\text {refurb }} . \\
C_{b}^{\text {refurb }}+b_{b}^{\text {hyd }} \cdot \\
\left.C_{b}^{\text {hyd }}\right)+\frac{1}{\varepsilon_{r, D}^{\text {tot }}} \sum_{\kappa} \sum_{t_{\kappa}} \sigma_{\kappa}\left(\sum_{b} \sum_{f} f_{f, t, b} \cdot P_{f}^{\text {fuel }}+\right. \\
\left(P_{t}^{\text {spot }}+P^{\text {grid }}+P^{\text {ret }}\right) \cdot\left(y_{t}^{\text {imp }}+\right. \\
\left.\left.\sum_{b} \sum_{e s t} y_{t, b, e s t}^{\text {imp }}\right)-P_{t}^{\text {spot }} \cdot y_{t}^{\text {exp }}\right)
\end{gathered}
$$

The $C$ are the cost associated with it. The capacity of technology $i$ in building $b$ is $x_{i, b}$, the associated discounted investment cost is $C_{i, b}^{\text {disc }}$ and the operation and maintenance cost is $C_{i, b}^{\text {maint }}$. The discount factor for the lifetime of the neighborhood with the discount rate $r$ is $\varepsilon_{r, D}^{t o t}$. The timestep inside cluster $\kappa$ is $t_{\kappa}$, and $\sigma_{\kappa}$ is the number of elements inside this cluster. The fuel consumption of technology burning fuel $f$ is $f_{f, t, b}$ and the cost of this fuel is $P_{f}^{\text {fuel }}$. $P_{t}^{\text {spot }}, P^{\text {grid }}$ and $P^{\text {ret }}$ are respectively the spot price of electricity, the grid tariff, and the retailer tariff. The import and export to the neighborhood are $y_{t}^{i m p}$ and $y_{t}^{e x p}$ while the import to battery est is $y_{t, b, e s t}^{i m p}$. The imports and exports of electricity are limited by the size of the grid connection.

To be a ZEN, the neighborhood needs to have net zero emissions in its lifetime. In the ZEN framework, we consider that the electricity exports from renewable sources in the neighborhood reduce the emissions in Norway by replacing some of the more carbon intensive generation. We call those avoided emissions "compensations" and the emission balance requires at least as much compensations as emissions. The constraint representing this is:

$$
\begin{gathered}
\phi_{t}^{C O_{2}, e} \sum_{\kappa} \sum_{t_{\kappa}} \sigma_{\kappa}\left(y_{t}^{i m p}+\right. \\
\left.\sum_{b} \sum_{e s t} y_{t, b, e s t}^{i m p}\right)+\sum_{\kappa} \sum_{t_{\kappa}} \sigma_{\kappa} \sum_{b} \sum_{f} \phi^{C O_{2}, f} . \\
f_{f, t, b} \leq \phi_{t}^{C O_{2}, e} \cdot \sum_{\kappa} \sum_{t_{\kappa}} \sigma_{\kappa}\left(\sum_{b} \sum_{e s t} \eta_{e s t}\right. \\
\left.y_{t, e s t, b}^{\text {exp }}+\sum_{b} \sum_{g} y_{t, g, b}^{\text {exp }}\right)
\end{gathered}
$$

In this equation, the $\mathrm{CO} 2$ factor for electricity is $\phi_{t}^{\mathrm{CO}_{2}, e}$ and the factor for other fuels is $\phi^{\mathrm{CO}_{2}, f}$. The efficiency of the battery is $\eta_{e s t}$.

The heat load balances are dependant of the investment in refurbishment, but the electric load is not affected by it:

$$
\begin{aligned}
& y_{t}^{i m p}+\sum_{b}\left(\sum_{e s t} y_{t, e s t, b}^{d c h} \cdot \eta_{e s t}+\sum_{g} g_{g, t, b}^{\text {selfc }}\right)= \\
& \sum_{b}\left(\sum_{e} d_{e, t, b}+E_{t, b}\right), \quad \forall t \\
& \sum_{q} q_{q, t, b}^{D H W}+\sum_{h s t}\left(\eta_{h s t} \cdot q_{t, h s t, b}^{D H W, d c h}-\right. \\
& \left.q_{t, h s t, b}^{D H W, c h}\right)+q_{t, h s t, b}^{H G, D H}=B_{b}^{r e f u r b} \cdot\left(H_{b, t}^{D H W}\right)+ \\
& \left(1-B_{b}^{\text {refurb }}\right) \cdot\left(\left(1-b_{b}^{\text {refurb }}\right) \cdot H_{b, t}^{D H W}+\right. \\
& \left.b_{b}^{\text {refurb }} \cdot H_{b, t}^{\text {refurb }, \text { DHW }}\right)+q_{t, b}^{\text {dump }}, \quad \forall b, t \\
& \sum_{q} q_{q, t, b}^{S H}+\sum_{h s t}\left(\eta_{h s t} \cdot q_{t, h s t, b}^{S H, d c h}-q_{t, h s t, b}^{S H, c h}\right)+ \\
& q_{t, h s t, b}^{H G, S H}=B_{b}^{\text {refurb }} \cdot\left(H_{b, t}^{S H}\right)+\left(1-B_{b}^{\text {refurb }}\right) \cdot \\
& \left(\left(1-b_{b}^{\text {refurb }}\right) \cdot H_{b, t}^{S H}+b_{b}^{\text {refurb }} .\right. \\
& \left.H_{b, t}^{r e f u r b, S H}\right), \quad \forall b, t
\end{aligned}
$$


The discharge from battery $b$ to the neighborhood is $y_{t, e s t, b}^{d c h}$ (similarly $q_{t, h s t, b}^{d c h}$ is the heat discharged from the heat storage $h s t$ to the neighborhood) and the electricity produced by technology $g$ directly consumed is $g_{g, t, b}^{\text {selfc }}$. The electricity consumption of heat producing technology $e$ is $d_{e, t, b}$ and the heat-independent electricity consumption is $E_{t, b}$. The heat produced by technology $q$ is $q_{q, t, b}$, distinguished between Space Heating (SH) and Domestic Hot Water (DHW). The heat demand is $H_{b, t} . B_{b}^{\text {refurb }}$ is a parameter indicating if building $b$ can be refurbished (1 meaning it cannot); and $b_{b}^{\text {refurb }}$ is a variable controlling the investment in refurbishment (1 meaning it chooses to refurbish).

If the optimization invests in a heating grid, technologies at the neighborhood level, i.e. larger scale technologies, become available.

$$
x_{i, P P} \leq X_{i}^{\max } \cdot b^{H G}
$$

The balance at the production plant (noted PP) where those technologies are located is then:

$$
\begin{gathered}
\sum_{q} q_{q, t, P P}+\sum_{h s t}\left(\eta_{h s t} \cdot q_{t, h s t, P P}^{d c h}-q_{t, h s t, P P}^{c h}\right)= \\
\sum_{b \backslash P P} q_{t, P P, b}^{\text {HGtrans }}+q_{t, P P}^{\text {dump }}, \quad \forall t
\end{gathered}
$$

Where $q_{t, P P, b}^{\text {HGtrans }}$ is the heat transferred in the heating grid from the production plant to building $b$ in timestep $t$.

The investment in $i$ is limited by the existing capacity $X_{i, b}^{\text {precap }}$, the minimum $\left(X_{i}^{\min }\right)$ and the maximum $\left(X_{i}^{\text {max }}\right)$ investment size:

$$
\begin{gathered}
X_{i, b}^{\text {precap }} \leq x_{i, b} \leq X_{i}^{\max }, \quad \forall i, b \\
X_{i}^{\text {min }} \cdot b_{i, b}^{i n v} \leq x_{i, b} \leq X_{i}^{\text {max }} \cdot b_{i, b}^{i n v}, \quad \forall i, b
\end{gathered}
$$

A binary $b_{i}^{i n v}$ is necessary for this semi-continuous formulation.

Some technologies also require a hydronic system to be installed (if they do, $B_{q}^{\text {hyd }}=1$ ).

$$
x_{q, b} \leq X_{q}^{\max } \cdot b_{b}^{\text {hyd }}, \quad \forall q, b
$$

Some technologies have different costs if the building is new, existing or is refurbished. In the case of buildings that can be refurbished, technologies in this category are represented as two investment options with different costs and the following constraint is needed to use the correct price. If the investment option is for existing buildings:

$$
x_{i, b} \leq X_{i}^{\max } \cdot\left(1-b_{b}^{\text {refurb }}\right), \quad \forall i, b
$$

If the investment option is for refurbished buildings:

$$
x_{i, b} \leq X_{i}^{\text {max }} \cdot b_{b}^{\text {refurb }}, \forall i, b
$$

The fuel or electricity used by technologies producing heat is:

$$
f_{f, t, b}=\frac{q_{f, t, b}}{\eta_{f}}, \forall f, t, b ; d_{e, t, b}=\frac{q_{e, t, b}}{\eta_{e}}, \quad \forall e, t, b
$$

where $\eta$ is the efficiency of the technology. The heat produced can fulfil SH and/or DHW depending on the technology; $B_{q}^{D H W}$ and $B_{q}^{S H}$ control which kind they can provide. An electric radiator for instance can only provide $\mathrm{SH}$. In addition, the hydronic system allows some technologies heating water to deliver $\mathrm{SH}$ in addition to the DHW when it is installed.

$$
\begin{gathered}
q_{f, t, b}=q_{q, t, b}^{D H W}+q_{q, t, b}^{S H}, \quad \forall q, t, b \\
q_{q, t, b}^{D H W} \leq M \cdot B_{q}^{D H W}, \quad \forall q, t, b \\
q_{q, t, b}^{S H} \leq M \cdot B_{q}^{S H}, \quad \forall q, t, b
\end{gathered}
$$

For CHPs, the efficiency is the one related to heat and the electricity production is obtained with the heat-topower ratio $\left(\alpha_{C H P}\right)$ :

$$
g_{f, t, b}=\frac{q_{f, t, b}}{\alpha_{f}}, \quad \forall C H P, t, b
$$

The solar technologies (solar thermal collector and PV panels) are modelled by their efficiency and the solar irradiance.

For heat pumps, the COP is used instead of the efficiency. It is calculated based on a polynomial fit of manufacturer's data and the difference between the supply and the source temperature. The max electric consumption $\left(P^{\text {in, } \max }\right)$ is also obtained in the same way. The supply temperature is $65^{\circ} \mathrm{C}$ for DHW and for $\mathrm{SH}$ it differs between recent (or refurbished) and old houses. If $B_{b}^{\text {refurb }}=1$, the heat pump is controlled in the following way:

$$
\begin{gathered}
d_{H P, t, b}^{S H}=\frac{q_{H P, t, b}^{S H}}{C O P_{H, t, b}^{S H}}, \quad \forall H P, t, b \\
d_{H P, t, b}^{D H W}=\frac{q_{H P, t, b}^{D H W}}{C O P_{H P, t, b}^{D H W}}, \quad \forall H P, t, b \\
\frac{d_{H P, t, b}^{S H}}{P_{H P, t, b}^{\text {in, max,SH}}}+\frac{d_{H P, t, b}^{D H W}}{P_{H P, t, b}^{i n, \max , D H W}} \leq x_{H P, b}, \quad \forall H P, t, b
\end{gathered}
$$

If $B_{b}^{\text {refurb }}=0$, the heat pump is affected by the decision to refurbish the building because of the impact on the supply temperature for the $\mathrm{SH}$. The heat production and electric consumption for $\mathrm{SH}$ is separated in two, using the notation $\mathrm{P}$ for passive and $\mathrm{NP}$ for not passive standard buildings.

$$
\begin{aligned}
& d_{H P, t, b}=d_{H P, t, b}^{S H, P}+d_{H P, t, b}^{S H, N P}+d_{H P, t, b}^{D H W} \\
& q_{H P, t, b}^{S H}=q_{H P, t, b}^{S H, P}+q_{H P, t, b}^{S H, N P} \\
& d_{H P, t, b}^{S H, P} \leq M \cdot b^{\text {refurb }} \\
& d_{H P, t, b}^{S H, N P} \leq M \cdot\left(1-b^{\text {refurb }}\right) \\
& \frac{d_{H P, t, b}^{S H, P}}{P_{H P, t, b}^{i n, m a x, S H, P}}+\frac{d_{H P, t, b}^{S H, N P}}{P_{H P, t, b}^{i n, m a x, S H, N P}}+\frac{d_{H P, t, b}^{D H W}}{P_{H P, t, b}^{i n, m a x, D H W}} \leq x_{H P, b}
\end{aligned}
$$

Constraints such as in Eq. (23) and (24) are called bigM constraints. $\mathrm{M}$ takes a very large value, not limiting the left-hand side of the equation if the binary is 1 and forcing it to 0 otherwise.

Some technologies can only be operated in a certain range of their nominal power. The part load limitation constraint ensures that the operation of those technologies is more realistic.

$$
\begin{gathered}
\overline{x_{l, t, b}} \leq X_{i}^{\max } \cdot o_{i, t, b} \\
x_{i, b}-X_{i}^{\max } \cdot\left(1-o_{i, t, b}\right) \leq \overline{x_{l, t, b}} \leq x_{i, b}
\end{gathered}
$$

Technology $i$, in $b$ is in operation in timestep $t$ when the binary $o_{i, t, b}$ is 1 . The semi-continuous variable of the effective capacity is represented by $\overline{x_{l, t, b}}$.

The heating grid is assumed to be radial and only fed by the central production plant, i.e. the buildings cannot feed heat into the heating grid.

$$
\begin{gathered}
q_{t, b}^{\text {HGused }}=\sum_{b^{\prime \prime}}\left(q_{t, b^{\prime \prime}, b}^{\text {HGtrans }}-Q_{b^{\prime \prime}, b}^{\text {HGloss }}\right)- \\
\sum_{b^{\prime}} q_{t, b, b \prime}^{\text {HGtrans }}, \forall t, b \\
q_{t, b}^{\text {HGused }} \geq 0, \forall t, b \\
q_{t, b^{\prime}, b}^{\text {HGtrans }} \leq \dot{Q}_{b^{\prime}, b}^{\text {max }, \text { pipe }}, \forall t, b, b^{\prime} \\
q_{t, b}^{\text {HGusedSH }} \leq M \cdot b_{b}^{\text {hyd }}, \forall t, b \\
q_{t, b}^{\text {HGusedDHW }} \leq M \cdot\left(b_{b}^{\text {hyd }}+B_{b}^{\text {DHWhyd }}\right), \forall t, b
\end{gathered}
$$


The size of the pipe limits the heat flow $\left(\dot{Q}_{b^{\prime}, b}^{\text {max,pipe }}\right)$ in the pipe, Eq. (31). The heat from the heating grid can only be used if a hydronic system is installed or for DHW in larger buildings if a hydronic system specifically for DHW already exists $\left(B_{b}^{\text {DHWhyd }}=1\right)$ as expressed by Eq. (32) and Eq. (33).

The operation of storage st (whether $\mathrm{SH}$, DHW, or electric) is modelled as follows:

$$
\begin{aligned}
& \forall \kappa, t_{\kappa} \in[1,23], s t, b \\
& v_{\kappa, t_{\kappa}, s t, b}^{\text {stor }}=v_{\kappa, t_{\kappa}-1, s t, b}^{\text {stor }}+\eta_{s t} \cdot q_{\kappa, t_{\kappa}, s t, b}^{c h} \\
& -q_{\kappa, t_{\kappa}, s t, b}^{d c h} \\
& \forall \kappa, t_{\kappa} \in[0,23], s t, b \\
& v_{\kappa, t_{\kappa, s t, b}}^{\text {stor }} \leq x_{s t, b} \\
& q_{\kappa, t_{\kappa}, s t, b}^{c h} \leq \dot{Q}_{s t}^{\max } ; q_{\kappa, t_{\kappa}, s t, b}^{d c h} \leq \dot{Q}_{s t}^{\max } \\
& \forall \kappa, s t, b \\
& v_{\kappa, 0, s t, b}^{\text {stor }}=v_{\kappa, 23, s t, b}^{\text {stor }}
\end{aligned}
$$

The storages have a daily operation (Eq. (37)) and a certain charging rate $\dot{Q}_{s t}^{\max }$.

The heat storage hst can only be charged and discharged if a hydronic system is installed in a similar way as in Eq. (32) and Eq. (33). Only technologies that heat up water can be used to charge the $\mathrm{SH}$ storage:

$$
\begin{gathered}
\sum_{h s t} q_{t, h s t, b}^{S H c h} \leq \sum_{q} q_{t, q, b}^{S H} \cdot B_{q}^{\text {hyd }}+ \\
q_{t, b}^{\text {HGusedSH }}, \forall t, b
\end{gathered}
$$

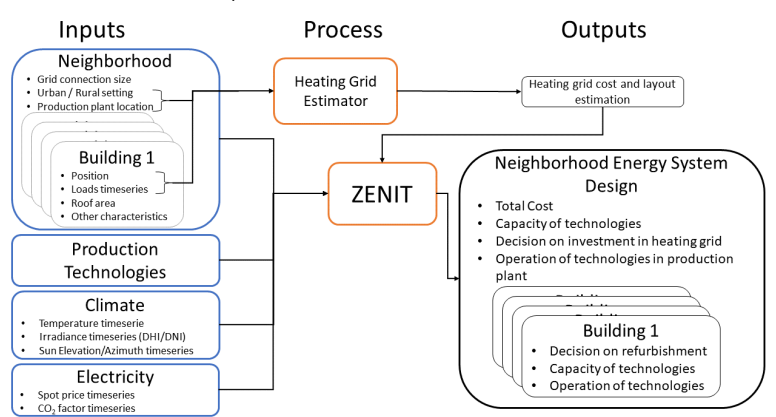

Fig.1. Representation of the inputs and outputs of the model

Fig.1 gives an overview of the different inputs and outputs of the model.

The next section presents the setup of the case study including more details about the inputs used.

\section{Case Study}

The neighborhood that is used in this model is a $250000 \mathrm{~m}^{2}$ ground floor area, $100000 \mathrm{~m}^{2}$ heated floor area. The floor area and share of each building type is based on the building mix of Oslo. The composition of the building mix and their ground area were obtained using GIS data from Oslo. In this case we consider seven types of buildings: houses (split in two blocks), apartments (split in two blocks), offices, shops, kindergarten, school, and nursing home. The loads timeseries of these buildings are obtained using the results from [13] and [14]. The loads of the buildings when refurbished are also obtained from those articles. The main data about the neighborhood is presented in Table 1 and the refurbishment costs in Table 2 . We assume a lifetime of 60 years for the neighorhood and a discount rate of $4 \%$.

Table 1. Characteristics of the building types in the neighborhood

\begin{tabular}{|l|l|l|l|l|l|}
\hline Type & $\begin{array}{l}\text { Area } \\
\left(\mathbf{m}^{2}\right)\end{array}$ & $\begin{array}{l}\text { Roof } \\
\text { Area }\end{array}$ & $\boldsymbol{B}_{\boldsymbol{b}}^{\text {reff }}$ & $\boldsymbol{B}_{\boldsymbol{b}}^{\text {hyd }}$ & $\boldsymbol{B}_{\boldsymbol{b}}^{\text {DHWhyd }}$ \\
\hline Houses1 & 13900 & 6950 & 0 & 0 & 0 \\
\hline Houses2 & 13900 & 6950 & 0 & 0 & 0 \\
\hline Apartments1 & 2205 & 4890 & 0 & 0 & 1 \\
\hline Apartment2 & 22005 & 4890 & 0 & 0 & 1 \\
\hline Offices & 18948 & 3158 & 0 & 0 & 0 \\
\hline Shops & 1230 & 1230 & 1 & 1 & 0 \\
\hline Kindergarten & 460 & 490 & 1 & 1 & 0 \\
\hline School & 5032 & 1677 & 0 & 0 & 1 \\
\hline Nursing Home & 1062 & 531 & 1 & 1 & 0 \\
\hline
\end{tabular}

Table 2. Refurbishment costs for the different buildings in euros

\begin{tabular}{|l|l|l|}
\hline Type & Refurbishment Cost & Hydronics Cost \\
\hline Houses 1 & $347500 €$ & $142500 €$ \\
\hline Houses 2 & $347500 €$ & $142500 €$ \\
\hline Apartments 1 & $550000 €$ & $150000 €$ \\
\hline Apartment2 & $550000 €$ & $150000 €$ \\
\hline Offices & $474000 €$ & $50000 €$ \\
\hline School & $12500 €$ & $25000 €$ \\
\hline
\end{tabular}

The hydronics costs are assumed based on various online sources and the refurbishment cost are derived from the numbers presented in [15] and set to around $25 € / \mathrm{m}^{2}$ of floor area. Those costs are assumptions to start with and we also investigate at which cost the optimization decides to refurbish in a sensitivity analysis.

The average load reduction in the $\mathrm{SH}$ loads of the buildings is $60 \%$. We assume that the DHW load is not affected by the refurbishment in this study.

Making realistic assumptions when it comes to the cost and the resulting load reduction is difficult and to apply this model to a real case, it would be beneficial to use it in combination with models such as the one presented in [10] and [11].

Several technologies are included in the study. At the building level, there are: solar panel, solar thermal collector, air-air heat pump, air-water heat pump, ground-source heat pump, bio boiler (wood logs or wood pellets), electric heater, electric boiler, biomethane boiler and gas, biogas and biomethane CHP. At the neighborhood level there are: CHP (biogas, wood chips or pellets), boiler (wood chips or pellets or electricity) and ground-source heat pump. The costs, efficiencies and other technical data about these technologies is taken from the Danish Energy Agency and can partly be found in [1].

The prices can be different depending on the status of the building (new, existing, or refurbished) and on the type of building (Apartment complex or single-family house).

All timeseries used correspond to year 2016. The timeserie for temperature comes from a measuring

\footnotetext{
$\$$ https://ens.dk/en/our-services/projections-and-models/technology-
} data 
station outside of Oslo ${ }^{\S}$. The spot prices come from Nordpool $^{* *}$ and the irradiance data from Solcast ${ }^{\dagger \dagger}$.

The model is run over one representative year clustered into 20 day-clusters to represent the lifetime. The binaries make this model hard to solve and the clustering counteracts that. More information on the clustering procedure can be found in [1]. If the technologies that are investigated do not require the use of the part load limitation, removing this constraint can yield significant improvements to the solving time and allow for the use of more clusters.

The model is implemented in Python and solved using Gurobi on a desktop with an Intel Core i5-7500 quadcore processor at $3.40 \mathrm{Ghz}$ and $24 \mathrm{~GB}$ of RAM but using only 3 threads.

We perform several cases. The first one is a direct application of the model presented earlier, the second is the same model except the binaries regarding the refurbishment and hydronics are linearized. In addition, we do a sensitivity analysis on the cost of the refurbishment.

\section{Results}

With a direct application of the model presented in this paper, we get the following investment:

At the production plant, $307 \mathrm{~kW}$ of biogas engine with heat recovery, $1245 \mathrm{kWh}$ batteries and $553 \mathrm{kWh}$ of heat storage.

For the building (aggregated results), $5713 \mathrm{~kW}$ of $\mathrm{PV}, 341 \mathrm{~kW}$ of solar thermal collectors (ST), $1.4 \mathrm{~kW}$ of air-air heat pump (HP), $664 \mathrm{~kW}$ of air-water heat pumps, $18 \mathrm{~kW}$ of electric water heater and $2116 \mathrm{kWh}$ of heat storage.

All the buildings where refurbishment is an option invested in a hydronic system, but none has chosen to refurbish. The total discounted cost for investment and operation of the neighborhood energy system is 16.124M€.

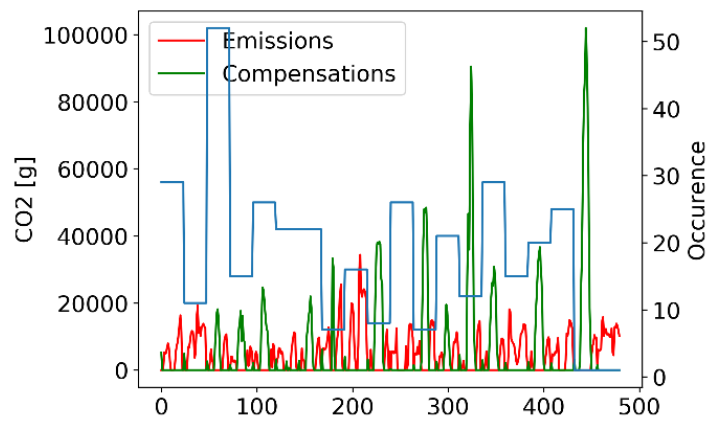

Fig. 2. Emissions and compensations of $\mathrm{CO} 2$ in the different clusters as well as the occurrence of the cluster (blue).

The emissions and compensation for each cluster as well as its occurrence in the year is presented on Fig. 2. The $\mathrm{CO} 2$ emissions are spread over all clusters while only a few clusters concentrate most of the compensations.

\footnotetext{
$\S$ https://Imt.nibio.no, Skjetten Station

** https://www.nordpoolgroup.com/Market-data1/\#/nordic/table
}

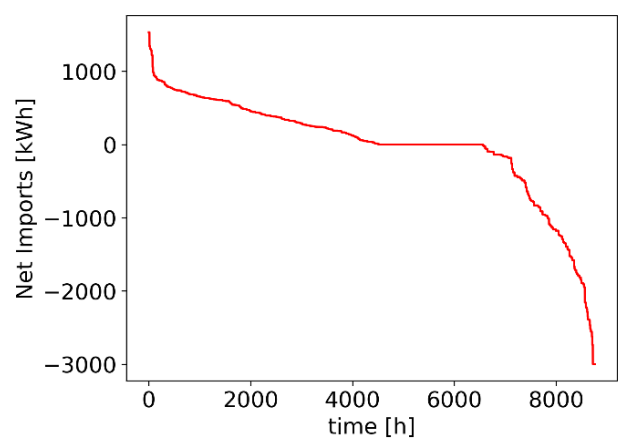

Fig. 3. Duration curve of net imports for the whole year reconstructed from the clusters

The duration curve of net import is shown on Fig. 3. The neighborhood is net importer for most of the year and the exports are concentrated in less than 2000 hours. The battery investment helps somewhat to achieve this by increasing the self-consumption and exporting when the $\mathrm{CO} 2$ factors are higher. The full potential of the battery is not fully used however due to the way it is modelled (24-hour operation).

The model is also used with the binaries for hydronics and refurbishment linearized. This gives quite different results:

At the neighborhood level, $5830 \mathrm{kWh}$ of battery.

In the buildings, $5661 \mathrm{~kW}$ of PV, $532 \mathrm{~kW}$ of ST, $1.47 \mathrm{~kW}$ air-air HP, $891 \mathrm{~kW}$ air-water HP, $110 \mathrm{~kW}$ waterwater $\mathrm{HP}, 40 \mathrm{~kW}$ wood $\log$ manual stoking boiler, $1.5 \mathrm{~kW}$ electric radiator and $10 \mathrm{~kW}$ electric boiler, with $643 \mathrm{kWh}$ SH storage and $1469 \mathrm{kWh}$ DHW storage.

The binaries for refurbishment and hydronics are linearized; the average value of the linearized binaries for refurbishment is 0.0331 and for hydronics it is 0.0657 .

The total discounted cost is $14.277 \mathrm{M} €$.

The linearized model gives quite different results than the one with binaries, both in terms of technologies and in terms of the hydronics investment.

The main difference in the energy system are that the heating grid and the biogas engine at the production plant are no longer chosen, and are replaced with other technologies inside buildings for the heat production as well as a bigger battery to allow for more of the PV to be exported. When there is no refurbishment much of the compensation originates from the biogas engine electricity production, that can follow the electricity $\mathrm{CO} 2$ factor timeseries, in particular the higher factors in the winter. With only PV, it is then necessary to use a bigger battery to maximize the compensation potential of the PV.

The solving time is longer for the linearized binaries version at around 47000 seconds versus 19000 seconds for the one with binaries, contrary to what one might expect.

One drawback of using the linearized version compared to the one with binaries has to do with the use of bigM constraints. Indeed, those constraint can be easily bypassed with a low value of the linearized binary due to the high value of M. For example, Eq. (10) and

${ }^{\dagger}$ https://solcast.com.au 
(32) illustrates this. In Eq. (32) a very low value is enough to disregard that equation due to the high value of $\mathrm{M}$; in Eq. (10), the value of the binary only needs to be set high enough to allow for the amount of capacity needed.

The linearized binary version of the model could be used to determine which buildings should be refurbished first or the proportion of a certain building type to be refurbished. However, the investment results might be distorted by the bigM constraints. Moreover, the actual values of the linearized binaries might not actually represent the proportion of buildings that should be refurbished. In our case, the refurbishment binaries have a very low value which indicates that the refurbishment itself is not profitable and the value is chosen only to affect the bigM constraints involving those binaries in order to profit from them without paying the total price.

In practice the investment sizes would depend on the nominal capacity of technologies available on the market. In addition, small investments like the air-air heat pump could be replaced or up-sized.

Since there is high uncertainty regarding a realistic pricing of the refurbishment, we investigate the price at which refurbishment starts to be chosen. The model using binaries is used with refurbishment prices of $75 \%$, $62.5 \%, 50 \%$ and $25 \%$ of the original refurbishment price (Table 2). At $75 \%$, the model still does not choose to refurbish, at $62.5 \%$ the refurbishment is done in just one

building, but from $50 \%$ and down, it does for all buildings.

We show the energy system for the $50 \%$ case to see how the system looks when refurbishment is chosen.

At the neighborhood level, $5754 \mathrm{kWh}$ of batteries.

In the buildings, $5643 \mathrm{~kW}$ of $\mathrm{PV}, 599 \mathrm{~kW}$ of $\mathrm{ST}$, $797 \mathrm{~kW}$ of air-water HP, $115 \mathrm{~kW}$ of water-water HP, $40 \mathrm{~kW}$ of manual stoking wood $\log$ boiler, $15 \mathrm{~kW}$ of electric boiler, $105 \mathrm{~kW}$ of biomethane boiler and $2420 \mathrm{kWh}$ of heat storage.

All buildings choose to invest in refurbishment and hydronic systems.

The total discounted cost is $16.007 \mathrm{M} €$.

Those results are quite similar to the results of the run with the linearized binaries. The amount of PV, ST and batteries are similar, and the main difference lie in the amount of air-water HP $(\sim 100 \mathrm{~kW})$ and heat storage $(\sim 300 \mathrm{kWh})$. This difference is explained by the lower $\mathrm{SH}$ demand when choosing refurbishment in the case with binaries.

\section{Conclusion}

In this paper, a model for investing in the energy system of ZENs, considering the refurbishment of buildings, the hydronics and their impacts on the model, is presented and used on a test case in Norway.

The results show that with the cost assumptions used, the refurbishment is not chosen, but hydronic systems are. The system relies mainly on PV, solar thermal collectors (ST), a biogas engine (connected to the buildings by a heating grid), a battery and heat pumps (HP) and heat storage. From $50 \%$ of the original refurbishment cost, refurbishment is chosen, and the system does not have a biogas engine and a heating grid anymore, but a much bigger battery and more heating technologies inside the buildings. With linearized binaries, the investments are similar to the case with $50 \%$ refurbishment cost, but the value of the linearized binaries cannot be used to indicate the share of buildings to refurbish.

This article has been written within the Research Center on Zero Emission Neighborhoods in Smart Cities (FME ZEN). The authors gratefully acknowledge the support from the ZEN partners and the Research Council of Norway.

The authors also thank Lillian Rokseth for providing the GIS data of Oslo.

\section{References}

[1] D. Pinel, "Clustering methods assessment for investment in zero emission neighborhoods' energy system," IJEPES, vol. 121, (2020).

[2] T. Capuder and P. Mancarella, "Technoeconomic and environmental modelling and optimization of flexible distributed multigeneration options," Energy, vol. 71, pp. 516 533, (2014).

[3] A. Fleischhacker, G. Lettner, D. Schwabeneder and $\mathrm{H}$. Auer, "Portfolio optimization of energy communities to meet reductions in costs and emissions," Energy, vol. 173, pp. 1092 - 1105, (2019).

[4] P. Gabrielli, M. Gazzani, E. Martelli and M. Mazzotti, "Optimal design of multi-energy systems with seasonal storage," Applied Energy, vol. 219, pp. 408 - 424, (2018).

[5] S. Mashayekh, M. Stadler, G. Cardoso and M. Heleno, "A mixed integer linear programming approach for optimal DER portfolio, sizing, and placement in multi-energy microgrids," Applied Energy, vol. 187, pp. 154 - 168, (2017).

[6] B. Morvaj, R. Evins and J. Carmeliet, "Optimising urban energy systems: Simultaneous system sizing, operation and district heating network layout," Energy, vol. 116, pp. 619 - 636, (2016).

[7] C. Weber and N. Shah, "Optimisation based design of a district energy system for an ecotown in the United Kingdom," Energy, vol. 36, pp. 1292 - 1308, (2011).

[8] M. Pavičević, T. Novosel, T. Pukšec and N. Duić, "Hourly optimization and sizing of district heating systems considering building refurbishment - Case study for the city of Zagreb," Energy, vol. 137, pp. 1264 - 1276, (2017).

[9] B. Fina, H. Auer and W. Friedl, "Profitability of active retrofitting of multi-apartment buildings: Building-attached/integrated photovoltaics with special consideration of different heating 
systems," Energy and Buildings, vol. 190, pp. 86-102, (2019).

[10] M. Fesanghary, S. Asadi and Z. W. Geem, "Design of low-emission and energy-efficient residential buildings using a multi-objective optimization algorithm," Building and Environment, vol. 49, pp. 245 - 250, (2012).

[11] W. Wang, R. Zmeureanu and H. Rivard, "Applying multi-objective genetic algorithms in green building design optimization," Building and Environment, vol. 40, no. 11, pp. 1512-1525, (2005).

[12] E. Antipova, D. Boer, G. Guillén-Gosálbez, L. F. Cabeza and L. Jiménez, "Multi-objective optimization coupled with life cycle assessment for retrofitting buildings," Energy and Buildings, vol. 82, pp. 92-99, (2014).

[13] K. B. Lindberg and G. Doorman, "Hourly load modelling of non-residential building stock," in Powertech, Grenoble, (2013).

[14] K. B. Lindberg, G. Doorman, J. E. Chacon and D. Fischer, "Hourly electricity load modelling of non-residential passive buildings in a nordic climate," in Powertech, Eindhoven, (2015).

[15] Y. Fan and X. Xia, "A Multi-objective Optimization Model for BuildingEnvelope Retrofit Planning," in ICAE, Energy Procedia, Abu Dhabi, (2015). 